\title{
Estrategias Metodológicas que Promueven la Inclusión en Educación Infantil, Primaria y Secundaria
}

\author{
Methodological Strategies that Promote Inclusion in Early \\ Childhood, Primary and Secondary Education
}

\author{
Inmaculada Orozco * \\ Anabel Moriña \\ Universidad de Sevilla, España
}

\begin{abstract}
En este artículo se analizan cuáles son las estrategias metodológicas que pone en práctica el profesorado español que desarrolla una pedagogía inclusiva en Educación Infantil, Primaria y Secundaria. Se ha llevado a cabo una investigación cualitativa mediante entrevistas semiestructuradas. Los participantes fueron 70 docentes de distintos centros educativos de la provincia de Sevilla. Los resultados muestran las semejanzas y diferencias que existen entre las tres etapas educativas en cuanto a las estrategias metodológicas utilizadas para facilitar el aprendizaje y participación de todo el alumnado. Se explican aquellas estrategias que utiliza este profesorado, para qué y cómo las emplean. En concreto, en este estudio se analizan siete estrategias metodológicas: trabajo por proyectos, aprendizaje cooperativo, tutoría entre iguales, aprendizaje dialógico, juego y gamificación, grupos interactivos, rincones, aprendizaje vivencial y Aprendizaje-Servicio. Las conclusiones revelan que estas estrategias metodológicas inclusivas ayudan a que otros colegas puedan nutrirse, mejorar su propia práctica y aprender de otras etapas educativas.
\end{abstract}

Descriptores: Pedagogía inclusiva; Estrategias metodológicas; Educación infantil; Educación primaria; Educación secundaria.

This article analyses the methodological strategies put into practice by Spanish teachers who develop an inclusive pedagogy in Early Childhood Education, Primary and Secondary Education. Qualitative research has been carried out using semistructured interviews. The participants were 70 teachers from different schools in the province of Seville. The results show the similarities and differences that exist between the three educational stages in terms of the methodological strategies used to facilitate learning and participation of all students. The strategies used by these teachers are explained, as well as what they do and how they use them. Specifically, this study analyses seven methodological strategies: project-based learning, cooperative learning, peer tutoring, dialogical learning, play and gamification, interactive groups, learning corners, experiential learning and service-learning. The conclusions reveal that the inclusive methodological strategies are an opportunity for other teachers to benefit themselves, improve their own practice and learning from other educational stages.

Keywords: Inclusive pedagogy; Methodological strategies; Early childhood education; Primary education; Secondary education.

*Contacto: iorozco@us.es

ISSN: 2254-3139

www.rinace.net/riejs/

revistas.uam.es/riejs
Recibido: $\quad 30$ de septiembre 2019

$1^{a}$ Evaluación: 15 de diciembre 2019

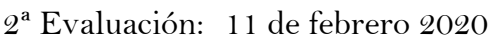

Aceptado: 28 de febrero 2020 


\section{Introducción}

Todos los alumnos ${ }^{1}$ tienen derecho a las mejores oportunidades para aprender, ser escuchados, participar y tener éxito (Ainscow, Slee y Best, 2019; Simón, Echeita y Sandoval, 2018; UNESCO, 2017). Sin embargo, para que este derecho se transforme en acción, es preciso que el profesorado quiera, se comprometa personalmente con las biografías personales de cada alumno, sea capaz de educar dando lo mejor de sí mismo y de hacerlo de una manera efectiva (Day, 2019; Herrera et al., 2018). Por tanto, para devolverle a la educación su sentido democrático y de justicia social, cada docente debe ser un agente de cambio intelectual crítico que vaya acorde con los discursos políticos y luche contra las prácticas de exclusión y los procesos de discriminación que han ido emergiendo a lo largo del tiempo (Arnaiz y Guirao, 2015; Belavi y Murillo, 2016; Santamaría y Stuardo, 2018).

Desde este planteamiento, las "dificultades de aprendizaje" no están en los estudiantes, sino en las barreras didácticas impuestas por el profesorado, es decir, en las estrategias metodológicas que ofrece a todos los estudiantes (López-Melero, 2016; Muntaner, Rosselló y De la Iglesia, 2016; Tjernberg y Mattson, 2014). Para lograr el aprendizaje y participación de todo el alumnado, los docentes deben repensar y transformar continuamente sus enfoques, estructuras organizativas y metodológicas para que cada persona perciba plenamente que es respetada porque no recibe una enseñanza diferenciada, sino ajustada a sus necesidades y peculiaridades (González-Gil, Martín-Pastor y Poy, 2019; Sharma y Jacobs, 2016; Spratt y Florian, 2015).

En definitiva, siguiendo la propuesta de Azorín y Sandoval (2019), con este trabajo, se pretende ofrecer a los centros y, en concreto, al profesorado de Educación Infantil, Primaria y Secundaria ${ }^{2}$ una serie de pautas y estrategias que le permita reflexionar sobre la práctica y emprender propuestas de cambio. El presente estudio tiene como objetivo conocer cuáles son las estrategias metodológicas que docentes de las distintas etapas educativas utilizan para lograr la participación de todo su alumnado en la vida del aula, sin excepción.

\section{Revisión de la literatura}

El primer paso para comprender cuál es el sustento que debe tener cualquier estrategia metodológica para considerarse inclusiva consiste en tener como premisa la pedagogía inclusiva, que en palabras de Florian (2014) hace referencia a un enfoque alternativo. Desde esta mirada, el profesorado responde a las diferencias individuales del alumnado, para evitar la exclusión de determinados estudiantes en las clases. Sabemos que todas ellas parten de la presencia, participación y progreso de todos los alumnos juntos en las misma actividades, experiencias y situaciones de aprendizaje (Black-Hawkins, Florian y Rouse, 2007; Muntaner, 2014). Este último autor manifiesta, además, que el estudiante debe estar en el centro del aprendizaje, sentirse seguro y conectar su aprendizaje con la realidad próxima.

\footnotetext{
${ }^{1}$ En este artículo se utiliza el masculino genérico como género gramatical no marcado.

2 Cuando hacemos referencia a Educación Secundaria incluimos a profesorado de Educación Secundaria Obligatoria, Bachillerato, Formación Profesional y Enseñanzas de Idiomas.
} 
Por otro lado, para Pujolàs (2002) las estrategias inclusivas solo serán inclusivas si estas incorporan tres requisitos: personalización de la enseñanza, autonomía del alumnado y estructuración cooperativa del aprendizaje. Este último también es apoyado por Crisol (2019) junto con las dinámicas de participación horizontal. Por tanto, las estrategias inclusivas trabajan con grupos heterogéneos y valoran los apoyos solo cuando están disponibles en el aula ordinaria y para todos (Muntaner et al., 2016). Además, todas ellas tienen como principios la globalización y la interdisciplinariedad, así como los espacios democráticos y de convivencia de la diversidad a través de asambleas, proyectos o problemas (López-Melero, 2018; Muntaner, 2014; Sales et al., 2018).

Por ejemplo, el proyecto "Comunidades de Aprendizaje", enmarcado dentro del Aprendizaje Dialógico, también está siendo un modelo referente en inclusión educativa porque demuestra que las buenas prácticas en las instituciones educativas dependen de las interacciones que se producen entre sus miembros (Díez-Palomar y Flecha, 2010). Estos autores las definen como un modelo educativo abierto a la comunidad que implica a todas las personas que influyen en el desarrollo de los estudiantes (familiares, profesorado, amigos, vecinos, asociaciones, voluntariado...) gracias a la puesta en práctica de actuaciones educativas de éxito: grupos interactivos, tertulias literarias dialógicas y bibliotecas tutorizadas entre otras.

Continuando desde esta perspectiva inclusiva, existe un estudio basado en la investigación-acción de Moliner, Sanahuja y Benet (2017) sobre las estrategias metodológicas inclusivas en las aulas ordinarias. En este trabajo se identifican estrategias que favorecen el aprendizaje y la participación de todo el alumnado: proyectos de trabajo (aprendizaje de manera significativa y globalizada dando respuesta a preguntas que nacen de las necesidades e intereses del alumnado) y enseñanza compartida, estrategias de aprendizaje cooperativo (trabajo en equipos reducidos para alcanzar objetivos comunes), tutoría entre iguales (creación de parejas de alumnos con una relación asimétrica con un objetivo común y compartido que se alcanza mediante una planificación docente), aprendizaje dialógico (aprendizaje a partir de las interacciones mediadas por un diálogo y relación igualitaria), grupos interactivos (multiplicación el aprendizaje y la convivencia de un grupo reducido y heterogéneo de alumnos al contar con la ayuda de personas adultas que facilitan las interacciones entre ellos), rincones (organización del aula por distintas zonas donde el alumnado va acudiendo libremente en función de sus intereses) y Aprendizaje-Servicio y comunitario (aprendizaje mientras se actúa sobre necesidades reales del entorno para mejorarlo).

También aparecen otras estrategias inclusivas como: Team Assisted Individualization (combina la enseñanza individual con el trabajo en equipo), tertulias dialógicas (construcción colectiva de significado y conocimiento en base al diálogo con todo el alumnado o personas participantes en la tertulia), autorregulación y regulación del aprendizaje, comisiones de apoyo entre compañeros y talleres.

En relación a la etapa de Educación Infantil, el estudio de Souto-Maninng (2017) incide en la importancia de la indagación, el autodescubrimiento y la resolución de problemas de la vida cotidiana. Las aportaciones de Henderson y Lasley (2014) abordan las actividades artísticas como la danza, la música, las artes visuales y el teatro. Harte (2010), por su parte, enfatiza el potencial del Trabajo por Proyectos. López-Melero (2018) apunta en esta misma dirección en el Proyecto Roma y todas sus estrategias didácticas, destacando especialmente los proyectos de investigación y la asamblea. Sargeant y Gillett-Swan 
(2019) defienden las prácticas que involucran a todos de manera activa y tienen en cuenta sus decisiones. Por último, Zhu, Li y Hsieh (2019) añaden el juego, las actividades cooperativas y las reuniones continuas con la familia.

En Educación Primaria, Spratt y Florian (2015) destacan los agrupamientos transitorios y el aprendizaje cooperativo. Traver-Martí, Moliner y Sales (2019) enfatizan en aquellas estrategias que se vinculan con las prácticas vinculadas al territorio y también mencionan el aprendizaje cooperativo, los proyectos de Aprendizaje-Servicio, las asambleas, el teatro y el uso de las redes sociales. Petrie, Devcich y Fitzgerald (2018), por su parte, destacan el papel activo del alumnado a través del diálogo y el feedback, así como el uso de herramientas pedagógicas variadas (mapas mentales, gráficos, collages o imágenes).

En Educación Secundaria, las contribuciones de Iglesias y otros (2013) dejan ver en sus resultados los beneficios de los grupos interactivos y las asambleas de aula para intercambiar puntos de vista entre los estudiantes. Del mismo modo, el estudio de Guerrero-Castañeda (2016), diseñado a partir de su experiencia sobre los obstáculos y los facilitadores para la participación de los adolescentes en las aulas, también expone que el aprendizaje cooperativo y los agrupamientos flexibles (división del alumnado en grupos definidos según capacidades y necesidades), la coenseñanza (dos docentes en el aula como parejas pedagógicas), el Diseño Universal para el Aprendizaje (ofrecer múltiples opciones de expresión, representación e implicación), así como los ambientes de aprendizaje son algunas estrategias metodológicas inclusivas.

En definitiva, son numerosos los estudios realizados sobre las estrategias focalizadas en el alumnado con Necesidades Educativas Especiales y etapas educativas y áreas de conocimiento concretas, pero no desde la óptica amplia de la diversidad y educar a todos los niños juntos (Ainscow, Slee y Best, 2019). Asimismo, aunque se detengan en las tres etapas abundan las investigaciones cuantitativas a través de cuestionarios o inventarios y sus resultados no han sido fruto de un contacto directo con los participantes para que estos puedan contar qué estrategia, cómo desarrollarla y por qué llevarla a la práctica (Sales et al., 2018). Por tanto, en esta investigación, pretendemos explorar cuáles son las estrategias que se están utilizando en diferentes etapas educativas a partir de las propuestas de Moliner, Sanahuja y Benet (2017), o si existen otras. Para ello, nos preguntamos: ¿Cuáles son las estrategias metodológicas inclusivas que emplea el profesorado de Educación Infantil, Primaria y Secundaria para el aprendizaje y participación de todo su alumnado?, ¿qué razones les impulsan a ponerlas en práctica?, y ¿cómo lo hacen?

\section{Método}

\section{Enfoque metodológico}

Los resultados de este artículo forman parte de un estudio más amplio, actualmente en ejecución, que analiza qué hace, cómo y por qué el profesorado desarrolla una pedagogía inclusiva en Educación Infantil, Primaria, Secundaria y Universidad ${ }^{3}$. En este trabajo nos centramos exclusivamente en la fase I de investigación y exploramos las estrategias

\footnotetext{
${ }^{3}$ Este trabajo se encuentra vinculado con el proyecto I+D+i "Pedagogía Inclusiva en la Universidad: Narrativas del Profesorado" [EDU2016-76587-R]
} 
metodológicas inclusivas que ponen en práctica docentes de todas las etapas educativas, a excepción de la universitaria.

En el estudio se utilizó una metodología cualitativa. En concreto, se llevó a cabo un proceso de inducción haciendo uso de los estudios multicaso (Stake, 2006), ya que no se trataba de generalizar los datos de cada caso, sino de interrelacionarlos, comprenderlos y transferirlos.

\section{Participantes}

En este trabajo los participantes fueron 70 docentes de las etapas de Educación Infantil, Primaria y Secundaria de centros educativos ubicados, en su mayoría, en zonas desfavorecidas con estudiantes en riesgo de exclusión social y vulnerabilidad.

Para seleccionar a los participantes se llevó a cabo un muestreo intencional por conveniencia y criterial. Los criterios que se formularon fueron seis: 1) docentes de Educación Infantil, Primaria, Secundaria, Bachillerato y Enseñanza Profesional que desarrollan una pedagogía inclusiva, 2) docentes de la provincia de Sevilla, 3) diversidad de edad, 4) diversidad de género, 5) diversidad de años de experiencia docente, y 6) disponibilidad en la participación y tiempo.

En primer lugar, se planificaron diferentes encuentros y reuniones presenciales con distintos asesores de los Centros de Profesorado de la provincia de Sevilla de las tres etapas, quienes ofrecieron contactos de docentes que estaban desarrollando una pedagogía inclusiva. Para garantizar la idoneidad de los participantes, algunas de las características que se les facilitó a dichos asesores para que decidieran tal elección fueron los siguientes: "facilita los procesos de aprendizaje, cree en las posibilidades de todo el alumnado, hace uso de estrategias de enseñanza activas, motiva a los estudiantes, etc.". Posteriormente, se recurrió a la técnica de muestreo no probabilística de bola de nieve (Cohen, Manion y Morrison, 2000), con el fin de contactar con colegas recomendados por los participantes ya entrevistados.

En Educación Infantil, fueron 23 docentes del segundo ciclo de infantil pertenecientes a trece escuelas públicas. De estos profesionales, se aprecia una prevalencia del género femenino, dado que 21 fueron maestras (91,3\%) y 2 maestros $(8,7 \%)$ con una edad media de 42 años. En cuanto a la experiencia docente, diez tenían entre diez y quince años de experiencia docente $(43,5 \%)$, cuatro entre 5 y $10(17,4 \%)$, cuatro entre 15 y $20(17,4 \%)$, tres entre 20 y $25(13 \%)$, uno entre 25 y $30(4,3 \%)$ y uno más de 30 años $(4,3 \%)$.

En Educación Primaria, participaron 25 docentes de diferentes especialidades y once escuelas públicas. De estos, 18 fueron mujeres (72\%) y 7 hombres (28\%) con una edad media de 44 años. Respecto a la experiencia docente, dos habían ejercido entre 5 y 10 años $(8 \%)$, seis entre 10 y 15 (24\%), siete entre 15 y 20 (28\%), dos entre 20 y $25(8 \%)$, cuatro entre 25 y $30(16 \%)$, tres entre 30 y 35 (12\%) y solo uno había desempeñado su profesión durante más de 35 años (4\%).

En Educación Secundaria, fueron 22 docentes de distintas especialidades y pertenecientes a trece institutos, de los cuales doce fueron públicos y uno privado. De estos docentes, 15 fueron mujeres $(68,2 \%)$ y 7 hombres $(31,8 \%)$ con una edad media de 47 años. En cuanto a su experiencia, siete habían ejercido entre 10 y 15 años $(31,8 \%)$, cuatro entre 15 y 20 $(18,2 \%)$, cuatro entre 20 y $25(18,2 \%)$, tres entre 25 y $30(13,6 \%)$ y solo tres más de treinta años $(13,6 \%)$. 


\section{Instrumentos de recogida de datos}

El instrumento que se elaboró para la fase 1 de investigación forma parte de una investigación más amplia sobre las cuatro dimensiones de la pedagogía inclusiva: creencias, conocimientos, diseños y acciones. En este artículo nos centraremos en la última dimensión.

Se trata de un guion de entrevista semiestructurada y en profundidad adaptado a cada una de las etapas educativas (desde Educación Infantil hasta Universidad), teniendo como referencia los estudios de Florian (2014), Gale, Mills y Cross (2017) y Rouse (2009).

\section{Trabajo de campo}

El registro de los datos comenzó en febrero de 2018 y continúa desarrollándose en el presente. Se hizo uso de una grabadora para elaborar la transcripción en primera persona de cada una de las entrevistas, las cuales tuvieron una duración promedio de dos horas y media. Algunas de las preguntas de investigación que abordamos durante los encuentros fueron: ¿Cuáles son las estrategias de enseñanza y aprendizaje que usted utiliza en clase?, ¿por qué?; de las estrategias que utiliza, ¿cuáles son las que ayudan a aumentar la participación y el logro de todos los estudiantes?, ¿por qué? Por último, todas las entrevistas fueron transcritas, y seguidamente, devueltas, negociadas y validadas con los profesionales a través de correo electrónico.

\section{Análisis de datos}

Se realizó un análisis cualitativo mediante un sistema de categorías y códigos inductivo manual que permitió dar sentido a los datos (Miles y Huberman, 1994). Posteriormente, para facilitar el manejo de la gran cantidad de datos recogidos, se insertó cada una de las entrevistas en el software MAXQDA 12, para un análisis riguroso y exhaustivo de la información.

Los códigos utilizados para esta investigación han sido dos: estrategias metodológicas y estrategias inclusivas fundamentales. De estos dos, aparecieron nueve subcódigos: trabajo por proyectos, aprendizaje cooperativo, tutoría entre iguales, aprendizaje dialógico, juego y gamificación, grupos interactivos, rincones, aprendizaje vivencial y AprendizajeServicio.

\section{Cuestiones éticas de la investigación}

A cada participante se le entregó un consentimiento informado, donde se le explicaba que su colaboración era voluntaria y su privacidad respetada desde el inicio hasta el final de la investigación. Sus nombres reales no se muestran en los resultados, sino que tan solo hay una abreviatura para representar cada etapa educativa $(\mathrm{I}=$ Infantil, $\mathrm{P}=$ Primaria, $\mathrm{S}=$ Secundaria).

\section{Resultados}

El profesorado de las etapas de Educación Infantil, Primaria y Secundaria tenía en común que no utilizaba una única estrategia metodológica, sino que eran varias y todas ellas se basaban en metodologías activas, participativas, personalizadas, globalizadas y constructivistas. Estas ponían al alumnado en el centro del aprendizaje, conllevaba la utilización de recursos diversos y eran aquellas que, según sus narrativas, mejores resultados le habían dado a lo largo de su experiencia y en su práctica diaria (trabajo por 
proyectos, aprendizaje cooperativo, tutorización entre iguales, aprendizaje dialógico, juego y gamificación, grupos interactivos, rincones, aprendizaje vivencial y AprendizajeServicio).

Los participantes de este estudio además de utilizar estas estrategias metodológicas coincidían en el uso de estrategias afectivas y emocionales: eran cercanos, resilientes y flexibles; acompañaban y facilitaban los procesos, confiaban en las capacidades de cada estudiante; se preocupaban por generar aprendizajes significativos y bienestar emocional; escuchaban y conocían los intereses y necesidades del alumnado y creaban un buen clima y cohesión grupal. Estas estrategias (junto a unas actitudes positivas frente a la diversidad), según expresaba cada participante desde su experiencia, habían resultado beneficiosas en la práctica porque aumentaba el aprendizaje y la participación del alumnado en la vida del aula. Para dar respuesta al objetivo de la investigación, a continuación, se muestran las semejanzas y diferencias que existen entre las tres etapas educativas en cuanto a las estrategias metodológicas utilizadas. Se explican aquellas estrategias que utiliza este profesorado, para qué y cómo las emplean. La secuencia que se muestra de las estrategias no se basa en la importancia de las estrategias o en ser más o menos inclusivas en las aulas, sino que se presentan siguiendo un orden de las más a menos utilizadas por los docentes.

\subsection{Trabajo por proyectos}

Esta estrategia fue la que gran parte del profesorado de las tres etapas educativas utilizaba, aunque en cada una de ellas la abordaban de manera distinta. En Educación Infantil, explicaban que esta estrategia metodológica tenía una aplicación en la vida, se ajustaba a las necesidades de los niños y permitía que todos se sintiesen representados ya que las temáticas que se abordaban en el aula no estaban impuestas por libros de texto o fases cerradas (según la teoría de Aprendizaje Basado en Proyectos), tampoco por los criterios docentes de acuerdo a lo estipulado en el currículo, sino que nacían de los intereses y preocupaciones reales y cotidianas del alumnado. De hecho, contaban que en algunos momentos surgía un solo proyecto, pero otras veces llevaban a cabo varios proyectos a la vez. Por ejemplo, algunos niños estaban interesados en averiguar en pequeños grupos o por parejas por qué no había plantas en el espacio mientras que otros solo habían decidido trabajar un problema que se abordaba en el grupo clase surgido tras una asamblea.

Por tanto, utilizaban los proyectos porque, sabían que, de esta manera, los niños encontraban sentido a lo que aprendían, dado que constantemente estaban reflexionando, haciendo e investigando junto a sus iguales. Los pasos que comentaban a la hora de desarrollar un proyecto eran fundamentalmente cuatro: escuchar, intentar resolver problemas en conjunto, facilitar información y reflexionar constantemente.

\footnotetext{
Ahora vamos a hacer un cohete, y aquí mis niños miden, reflexionan sobre la medida (cuántos cabemos, cómo tenemos que ponernos, las posiciones...) y todo esto se puede extrapolar a otras situaciones. Ta te digo, el tema surge de ellos, de sus dudas e intereses, y entre todos intentamos dar respuesta a eso que quieren conocer, pero siempre argumentándolo. Poco a poco ellos van haciendo sus hipótesis, y yo lo que pretendo es que esas hipótesis se confronten. Ahora, por ejemplo, estamos con el espacio y yo no quiero que sepan la teoría de qué es una estrella como me marcaría un libro, yo voy por sus intereses. Entonces, si ellos quieren jugar con un cohete, nos planteamos cómo podemos construirlo. (I16)
}

En Educación Primaria, una gran parte del profesorado insistió en el componente emocional de esta estrategia y solía trabajar más los proyectos como tareas integradas en 
grupo y con roles cooperativos. Aunque algunos participantes estaban iniciándose en la formación y reclamaban el poco tiempo que disponían en su jornada, ellos hicieron hincapié en esta agrupación ya que de esta manera cada uno podía ofrecer, a su ritmo, lo bueno que tenía. De hecho, también incorporaban la utilización de distintos espacios para su desarrollo (pasillos, aula de informática, otras clases...) así como la formación y participación con la familia. Por otro lado, insistieron en las tareas de exposición, una vez que se daba por acabado el proyecto, que podía ser en la misma clase, a otras aulas o al propio centro. Además, coincidían en la existencia de varios proyectos dentro del mismo aula y proyectos a nivel de centro por comisiones internas.

Al inicio de cada proyecto explicaban que este se tejía a partir de preguntas (algunas lanzadas por los propios docentes) donde los niños iban creando su propio itinerario de aprendizaje. La recogida de información de la temática solían hacerla a partir de encuestas, cuestionarios o solicitando ayuda y materiales a la familia u otros agentes externos.

Cuando trabajas por proyectos, investigación o tareas, lo importante es que el alumno exprese qué conoce del tema, qué quiere conocer y cómo lo vamos a investigar. Entonces, yo siempre parto de preguntar al alumnado. Es importante hacer buenas preguntas para que el alumno se desarrolle, investigue y haga. (P14)

En Educación Secundaria, el sentido de los proyectos parece esfumarse y suelen llevarlo a la práctica como resolución de problemas en pequeños grupos de trabajo para la evaluación de competencias. De hecho, estos proyectos suelen estar diseñados previamente por el profesorado según lo establecido en el currículum de la etapa, la familia no forma parte de los mismos y el alumnado sugiere algunas actividades, selecciona cómo desarrollarlo y lo transforma en un producto personal.

Cuando yo construyo proyectos en Tecnología con mucha frecuencia les suelo decir cuál es el modelo de proyecto y que ellos elijan la estructura que quieran (un puente, una grúa, una torre, un cacharrito de feria, una caseta...). Yo intento diseñar teniendo en cuenta que hay que dejar esa libertad para fomentar la autonomía bajo cierta responsabilidad. Luego, voy viendo que eso está haciéndose bien. Si una persona quiere hacer mejor una noria que un puente, ¿por qué voy a obligar que haga una noria? No tiene sentido. (S5)

\subsection{Aprendizaje cooperativo}

El profesorado de Educación Infantil mencionaba que utilizaba el aprendizaje cooperativo en sus prácticas. Sin embargo, a lo largo de los encuentros no aparecieron qué técnicas empleaban, sino tan solo manifestaban que tendían a realizar grupos. Estos equipos eran heterogéneos y cada miembro desempeñaba un rol diferente dentro de los mismos.

\footnotetext{
Se establecen roles y van rotando. Cuando se hace puesta común hay uno que se encarga de llevar la voz cantante que es el portavoz y este va rotando. Luego está el rol que anota, también va rotando... (I14)
}

En cambio, en Educación Primaria se podía apreciar que una mayoría de los profesionales utilizaban una variedad de técnicas, entre las que destacaban el saco de dudas, el folio giratorio, lápices al centro y el rompecabezas. Reconocieron que el aprendizaje cooperativo era una de las que más éxito tenía en sus prácticas de cara a la inclusión educativa porque fomentaba el respeto, la solidaridad y el diálogo. Además, argumentaban que, sin la participación de una de las personas del equipo, el grupo no tenía éxito.

Con el cooperativo haces que todos sean miembros imprescindibles del grupo, además la participación está asegurada. También utilizo mucho la del folio giratorio porque creo que es bastante sencilla, se puede adaptar a cualquier nivel. (P1) 
En Educación Secundaria aparecía con más claridad cómo vivenciaban el aprendizaje cooperativo. Una gran parte del profesorado argumentaba que era fundamental para dar responsabilidades a los estudiantes. De hecho, para garantizar una adecuada gestión del aula formaban grupos de trabajo heterogéneos, donde cada estudiante tenía un rol establecido de los mismos (guardar el silencio, manejo del tiempo, reparto de material...). Según explicaban, se hacía un reparto de tareas en equipos que iban rotando cada mes (grupos bases), donde cada uno de ellos tenía un nombre y cada miembro un rol. Este papel se asignaba a la persona que tenía esa aptitud para aprovechar los valores y hacerlos visibles en grupo. También estos roles iban cambiando para que todos los componentes pudieran vivenciarlo.

Entre las técnicas que utilizaban sobresalían el folio giratorio, lápices al centro, rompecabezas y cabezas numeradas, así como la dinámica de bola de nieve.

\begin{abstract}
A ver, una de las preguntas que le hago cuando les enseño Ciencias Naturales es: ¿̨el agua de los ríos es salada o dulce? Todos me dicen que el agua de los ríos es dulce y la de la mar salada, pero yo les pregunto por qué para que debatan y busquen razones en grupo. Así, con cabezas numeradas, pues todos participan. Luego, también puedo hacer un folio giratorio para que todos tengan que participar. (S5)
\end{abstract}

\title{
4.3. Tutoría entre iguales
}

Solo unos pocos docentes de Educación Infantil narraron la utilidad que tenía en su práctica la tutorización entre iguales. Esta estrategia era clave para hacer visible los puntos fuertes que tenía cada niño y crear una cohesión de grupo. Solían crear parejas de alumnos en las que uno asumía el rol de tutor y otro de tutorado con el fin de conseguir un objetivo común. Para el profesorado, además, esta estrategia era esencial para que el grupo tuviera una variedad de referentes distintos al adulto, también para originar la ayuda y la empatía en el aula. Al mismo tiempo, era una oportunidad para incrementar la autonomía y la motivación de todos por el aprendizaje.

Delegar también en los mismos niños la ayuda, tú no puedes hacer todo. Yo siempre fomento la ayuda a los demás porque muchas veces ellos dicen "no puedo, maestra" o "ponme esto". A esto, les suelo decir: "dile a no sé quién que te ayude". (I2)

En Educación Primaria casi todo el profesorado hacía un uso cotidiano de esta estrategia, es más, los propios estudiantes estaban sensibilizados con la dinámica. Al principio, según contaban, era un proceso complejo, pero conforme se desarrollaba el curso apreciaban que el alumnado se ayudaba con naturalidad y aprendía más que con la ayuda del docente. Por ejemplo, narraron que cuando mandaban una tarea, ellos eran meramente guías y aquel estudiante que acabara primero se dirigía hacia otro para conseguir resolverla en compañía.

En mi clase todos juegan a la vez, y cualquiera en un determinado momento le puede enseñar cosas a otro. Esto es lo primordial. Cuando a un alumno se le da bien el fútbol, enseña a otro, pero ese otro sabe saltar a la comba y también puede ayudar. (P9).

En Educación Secundaria, numerosos profesionales solían recurrir a esta estrategia. Según explicaban, estos estudiantes solían estar formados para que los contenidos realmente fueran afianzados y la ayuda efectiva. Además, este apoyo no solo iba destinado a un alumno en concreto, sino que se convertía en un recurso más para el docente en las clases.

Si veo que no estoy pudiendo de una manera, lo intento de otra y si no puedo, pues le pido ayuda a uno para que le eche una mano. Muchas veces no soy yo el que da con la forma con la que aprende, sino que con el trabajo colaborativo lo consiguen. (S9) 


\subsection{Aprendizaje dialógico}

En las distintas etapas se aprecia que aprender es una actividad social mediada por el lenguaje. En otras palabras, entienden que el aprendizaje depende de las interacciones y no de la individualidad. Aunque en cada etapa llevan a cabo estrategias y técnicas distintas (asambleas, debates, coloquios...), todos se basan en los principios de la capacidad universal del lenguaje de las personas y el diálogo igualitario que defiende el aprendizaje dialógico.

En Educación Infantil, casi todos los profesionales usaban con frecuencia las asambleas. Esta era una de las estrategias que consideraban clave para la participación de todos. Empleaban esta estrategia porque sabían que les permitía realizar encuentros vitales de reconocimiento y escucha donde se construía un proyecto de aula que partía de las necesidades y preguntas del grupo. Además, cada uno podía mostrarse tal como era, se tejían incertidumbres, se escuchaba y empatizaba. La función docente en este espacio no era la de dirigir la conversación, sino acompañar las propuestas e inquietudes de todos y cada uno de los niños.

En las asambleas yo lo que quiero es que siempre haya un para qué. A veces el para qué simplemente es porque estamos disfrutando, otras veces es porque queremos construir algo o ir a un determinado sitio. En función de ese itinerario que me van haciendo ellos, yo busco esas estrategias. To también traigo cosas al aula y estas dan pie para que ellos hablen, se hagan nuevas preguntas y sigan investigando. (I12)

En Educación Primaria, una gran parte del profesorado potenciaba la interacción oral mediante las asambleas. En estos espacios de encuentros primaba la conversación cultural (hablar acerca de lo que ocurre en el mundo y entre las personas que forman parte). Principalmente resaltaron la importancia de dar voz a los estudiantes, escuchar y estar atentos a sus intereses para diseñar en consonancia a sus propuestas. Al hilo de ello, la estrategia que sobresalió fue dar preguntas al alumnado y conseguir, en un ambiente distendido, que ellos hicieran y se hicieran a sí mismos cuestiones que generaran curiosidad por el aprendizaje.

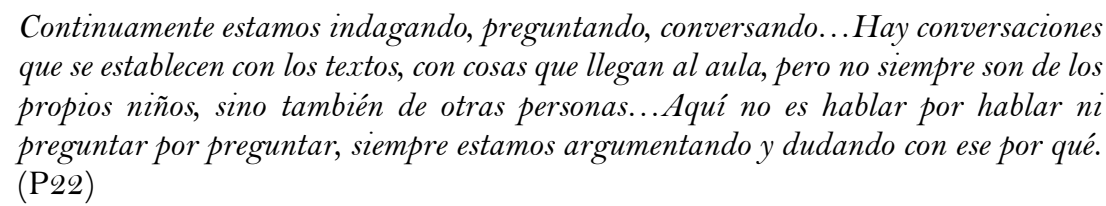

En Educación Secundaria también una mayoría del profesorado recurría a las asambleas, el método socrático, los debates y los coloquios. A través de estas estrategias desarrollaban gran parte de sus clases, las cuales comenzaban con preguntas escritas en una pizarra o lanzadas en voz alta. Estos momentos permitían que cada estudiante mostrara sin miedo sus dudas, intereses e ideas respecto a cualquier contenido de la asignatura. Todas las cuestiones y aportaciones del grupo eran valiosas para construir el aprendizaje juntos. Para ellos también era clave dejar claras las normas de convivencia como el respeto al turno de palabra y las opiniones de cada uno.

Cuando llegamos siempre se empieza modo asamblea. Por ejemplo, si estamos trabajando Platón o Aristóteles pongo preguntas iniciales: ¿̇a qué llamamos bueno?, ¿qué es el amor?, ¿qué es la felicidad? A partir de ahí empezamos, ellos comienzan a hablar y cogen confianza. Yo siempre estoy haciendo todo lo posible para que saquen sus argumentos. (S13) 


\subsection{Juego y gamificación}

En Educación Infantil, los docentes explicaron que utilizaban el juego como estrategia metodológica. Sin embargo, estos comentaban que la gran parte del día la dedicaban a estar junto al alumnado jugando a los juegos de mesas, cooperativos, de patio, libres y dirigidos. La razón que daban era porque de esta manera los niños se desinhibían, eran ellos mismos y comenzaban a confiar en el docente. Además, el componente lúdico, según contaban, favorecía la motivación por el conocimiento, la socialización entre iguales y la autonomía de cada uno. Gracias al juego nadie quedaba fuera del proceso educativo y era posible respetar los ritmos de aprendizaje.

\section{El juego hace que todos quieran participar. No es lo mismo contar los números o contar en un papel que ponernos, como hacemos, libremente a experimentar con animales. Dejamos que poco a poco comiencen a hacer series, jugar con ellos, quitarlos, ver cuántos hay...(I17)}

En Educación Primaria, una pequeña proporción del profesorado hacía uso del juego, pero también apostaba por la gamificación para generar motivación en el aprendizaje y establecer relaciones con la familia. En algunos casos, gracias a la formación que habían recibido en Neurociencia, utilizaban juegos de equilibrio y ritmo a mitad de la clase para hacer descansos y aumentar la concentración a la vez que disfrutaban con sus compañeros. Otros participantes, empleaban el juego para repasar los contenidos aprendidos. Por ejemplo, una maestra pidió a los estudiantes que, por parejas o tríos diseñaran su propio rosco de Pasapalabra (programa de TV) sobre los temas que estaban trabajando en las asignaturas de Naturales y Lengua. Finalmente, todos los roscos fueron subidos a una plataforma virtual para que en grupos respondieran a ellas.

En el caso de la Gamificación, solían utilizar aplicaciones tecnológicas como Plickers, Classcraft, Story Bird y Faceclass para hacer exámenes, mandar y corregir ejercicios y comunicarse con la familia. Por ejemplo, una maestra relató que Classcraft era una de las que más usaba porque le permitía dar feedback al alumnado mediante la evolución de un personaje virtual que se generaba a través de bonos y retos.

\section{A través de Classcraft ellos tienen unos personajes en una plataforma con unas tareas que tienen que hacer. Tienen una serie de puntos que yo les voy dando por trabajar de forma individual, por trabajar de forma cooperativa. Con estos puntos que van ganando pueden ir mejorando su personaje y hacer que evolucione. Ellos están todo el día mirando cuántos puntos llevan, cuántos poderes tienen, cómo pueden mejorar para que el personaje cambie de ropa...(P 1$)$}

En Educación Secundaria, una gran parte del profesorado planteaba concursos, ofrecían bonos y generaban retos y, al igual que en Educación Primaria, pedían que diseñaran sus propios juegos. Por ejemplo, para que todos asimilaran mejor los tiempos verbales en inglés, pedían que cada grupo elaborara un tablero con preguntas para jugar con un dado, teniendo como referentes juegos tradicionales como la Oca o el Bingo. Estos juegos iban rotando por los distintos equipos, de manera que se creaba un buen clima de aula porque todos disfrutaban de juegos que ellos mismos habían hecho y aprendían a autoevaluar y valorar el trabajo de los demás.

Otros participantes empleaban aplicaciones web lúdicas para evaluar los conocimientos previos y reforzar los contenidos de la asignatura. De esta manera, todos disfrutaban y afianzaban mejor los aprendizajes. Por ejemplo, una profesora de francés utilizaba Jeopardy para simular un concurso de televisión. 
To utilizo mucho Jeopardy. Se trata de una aplicación donde tú pones las preguntas que quieras. To hago así concursos con ellos. Esto se hace en la pizarra digital y tú metes lo jugadores que quieras. To me llevo un reloj de arena y les digo que elijan un portavoz por grupo. A ver, aquí hay un trabajo previo porque tú tienes que elaborar las respuestas. De todos modos, imagínate esto dentro de una clase, ellos disfrutan. En la pizarra aparece como ellos se van organizando según si ganan o pierden, pero tienen claro que un equipo es un equipo. Aquí todos entran y no hay diferencia. (S16).

\title{
4.6. Grupos interactivos
}

Esta estrategia metodológica no apareció en Secundaria y solo la desarrollaban docentes de Educación Infantil y Primaria que pertenecían a centros que eran Comunidades de Aprendizaje. En el caso de Educación Infantil, los participantes narraron que a través de ella fomentaban el aprendizaje cooperativo y las interacciones, al mismo tiempo que podían profundizar y descubrir cómo aprendía mejor el alumnado y cómo ayudarle en su proyecto vital.

En concreto, en esta etapa se preocupaban especialmente por la participación de la familia, la formaban para la monitorización de las actividades y se coordinaban semanalmente para la elaboración de materiales.

\begin{abstract}
Ahora, en cada grupo se desarrolla una actividad, pero se supone que esas actividades tienen que ser sobre contenidos que los niños ya conocen, que no es algo nuevo del momento. Entonces, se les plantea una actividad y tienen que ponerse de acuerdo y realizarla entre todos. El papel del adulto no es dar las respuestas, sino dinamizar, hacer que todo vaya fluido, y que, si el grupo se atasca, ofrecer un empujoncito para que sigan. (I20)
\end{abstract}

En Educación Primaria, también había docentes que apostaban por esta estrategia. La razón principal era porque de esta manera todos aprendían de todos y todas las aportaciones se respetaban de cara a construir una respuesta o solución común. De igual modo, consideraban a las familias como agentes imprescindibles para que la actuación fuera plenamente exitosa. Ellos solían realizarlo una vez a la semana y, de igual modo, se reunían con el resto de adultos ya mencionados en la etapa de Infantil para planificar las sesiones. Además, insistían en los grupos heterogéneos, ya que mientras más diversos fueran, más aprendizaje habría.

\begin{abstract}
Recurro a los grupos interactivos y cuando lo hacemos, son grupos de no más de cuatro o cinco alumnos con idea de que todos puedan participar. Luego, por supuesto variado en cuanto a sexo, características, necesidades..., en fin, mientras más variado posible, mejor. (I23)
\end{abstract}

\subsection{Rincones}

Esta estrategia metodológica únicamente era utilizada por el profesorado de Educación Infantil. Se trataba de rincones de juego libre y rotatorios, aunque había algunos dirigidos para una determinada actividad cuando se quería que todos trabajasen a lo largo de un tiempo. En la mayoría de las ocasiones solían tener una zona dedicada a la asamblea, un rincón de las construcciones, de juego simbólico, expresión plástica, lenguaje, naturaleza y lógico-matemática. A cada rincón iba un mínimo de cinco o seis niños, pero estos eran flexibles y se adaptaban a las necesidades, los ritmos e intereses. Estos espacios, a su vez, permitían a los participantes aprender cómo aprenden las personas y ofrecían al alumnado contenidos funcionales para su vida.

El juego por rincones lo utilizo para que cada uno pueda un poco ir a su ritmo, elegir y centrarse en el aspecto que más quiere. Lo que más hace es que todos tengan esa cabida porque cada uno está haciendo su actividad, algunos son más libres, otros más 
dirigidos, pero cada uno va a su ritmo. Esto te permite estar en un rincón con un grupo, irlos llamando o atender de uno en uno. (I15)

\subsection{Aprendizaje vivencial}

Solo algunos docentes de Educación Secundaria tendían a realizar actividades que estuviesen relacionadas con la realidad que vivían sus estudiantes para que encontraran sentido al aprendizaje y sintieran que podían aplicarlo. Por ejemplo, en el grado de Formación Profesional de cocina hicieron uso del programa de Mastercheff; para trabajar la expresión oral en la asignatura de Lengua simularon una entrevista de trabajo y en inglés hicieron un mercadillo bilingüe porque la mayoría de las familias trabajan en los mercadillos del barrio.

Me he dado cuenta que los contenidos tienen que conectar con su realidad, su entorno, su familia, sus aficiones... Esto le abre a la participación y a que se interesen por los contenidos. En el momento en el que ellos se familiarizan con los compañeros, conmigo y ven que la enseñanza tiene conexión con la realidad, pues el proceso de aprendizaje es más rápido. ( $\mathrm{S} 12)$

\subsection{Aprendizaje-Servicio}

Aunque la mayoría de los docentes de las tres etapas conocían esta estrategia metodológica y hablaban de su importancia en las escuelas y los institutos, solo tres participantes de Educación Primaria lo llevaban a cabo en su práctica debido a su motivación y el apoyo recibido por el equipo directivo. Una maestra contaba que los contenidos de su asignatura eran complejos de entender y que su labor era hacerlos más sencillos y vivenciales. No obstante, para que los aprendizajes tuvieran una repercusión social utilizó esta estrategia para montar una campaña de transfusión de sangre en su propio centro. A la vez que permitía que todo el alumnado participara y se sintiera protagonista en grupos cooperativos, contribuyó junto a sus estudiantes a la mejora de la vida de otras personas.

En sexto hay aspectos, por ejemplo, de Ciencias que son bastantes complicados. Entonces, en el primer trimestre planteamos un proyecto que estaba basado en las donaciones de sangre. Era un Proyecto de Aprendizaje-Servicio, donde nosotros fuimos al centro de transfusiones sanguíneas para pedirle que vinieran al cole, hicimos y pegamos carteles por las tiendas, hicimos nuestras donaciones, vinieron a darnos charlas, entrevistamos a personas con enfermedades...(P1)

\section{Discusión y conclusiones}

El profesorado de Educación Infantil, Primaria y Secundaria que ha participado en este estudio hace uso de estrategias metodológicas múltiples que sitúan al alumnado en el centro del aprendizaje y al docente en acompañante de cada proceso único, considerando que si no es así la educación no tiene sentido ni es para todas las personas. Se ha podido apreciar que algunas estrategias coincidían entre etapas, pero otras iban tomando otro carácter y significado en función de estas. Esto nos lleva a pensar que lo que hace que una estrategia sea inclusiva no es la estrategia en sí, si no el uso que de esta hace el docente y los ajustes razonables que lleva a cabo en un grupo humano que siempre tendrá necesidades y será diverso. Por tanto, las estrategias metodológicas para que sean inclusivas pueden depender del compromiso docente y cómo este vive su profesión, los apoyos que recibe por parte de los profesionales de su entorno inmediato, u otras circunstancias (Day, 2019; Herrera et al., 2018). El trabajo por proyectos, la tutoría entre iguales, el aprendizaje cooperativo, el juego y el aprendizaje dialógico han sido cuatro 
estrategias que han sobresalido y pueden definirse como acciones justas para conseguir una institución y aula más inclusivas (Crisol, 2019; Guerrero-Castañeda, 2016; Harte, 2010; López-Melero, 2018; Sales et al., 2018; Zhu, Li y Hsieh, 2019). Por tanto, dado que el apoyo humano es el más valioso que existe, sería recomendable que los propios equipos directivos y el profesorado aunaran fuerzas y demandaran formación en esta línea, organizaran reuniones interciclos semanales y promovieran proyectos integrados de distintas asignaturas en esta dirección. Esto sería una oportunidad para dialogar, intercambiar experiencias, disfrutar, aprender a escuchar y compartir humildemente dudas e inquietudes.

No obstante, en relación a los proyectos de Trabajo, el docente de Educación Secundaria debería aprender y dejarse contagiar por el buen hacer de los profesionales de otras etapas. El hecho de implementar los proyectos no debe ser visto como una utopía ni una pérdida de tiempo en determinados niveles, sino una vía para facilitar el aprendizaje, incentivar la investigación y la curiosidad de los estudiantes, así como fomentar las relaciones sociales en las clases. Solo se trata de valorar que el conocimiento se construye a partir de las interacciones con cada una de las personas que conforman el grupo.

Por otra parte, como se ha podido evidenciar en este estudio, el aprendizaje cooperativo es una de las estrategias que están ocultas en las primeras edades. En este caso, este resultado obtenido se contradice con los hallazgos de Zhu, Li y Hsieh (2019) porque los docentes de infantil no han nombrado ninguna técnica cooperativa. De esta manera, sería interesante que el profesorado recibiera formación (bien por parte de los centros de formación o, por ejemplo, asistiendo a observar clases de docentes que estén practicándola) de cara a eliminar barreras didácticas e incorporar estas estrategias en sus aulas.

Esta misma acción de formarse con otros profesionales se podría llevar a cabo con los rincones de trabajo de Educación Primaria y Secundaria, siendo esta vez los profesionales de infantil quienes compartirían su experiencia. Los tiempos y espacios en estas etapas suelen dificultar una organización inclusiva que respete los ritmos de aprendizaje del alumnado, y el trabajo por rincones sería una estrategia que podría dar respuesta a las necesidades de cada uno. Por tanto, sería imprescindible que el claustro de los centros reflexionara sobre la organización de los espacios y las oportunidades que se ofrecen al alumnado para que pudieran aprender libremente, en zonas distintas habilitadas y con recursos variados donde experimentar y aprender con los iguales de acuerdo a sus necesidades.

Por otro lado, coincidimos con la propuesta de Moliner, Sanahuja y Benet (2017) en cuanto a la utilización de los grupos interactivos como estrategias metodológicas inclusivas, pero también añadimos que en nuestro estudio tan solo docentes de centros que son Comunidades de Aprendizaje y en las etapas de Educación Infantil y Primaria fomentan su puesta en práctica. La escasa participación de la familia y otros agentes sociales (como voluntariado u ONGs) en las aulas de secundaria podrían solventarse o incrementar con esta estrategia metodológica, independientemente del centro al que pertenezcan. En la formación inicial de profesorado, sería recomendable que los futuros docentes tuvieran contacto con esta realidad a través de las prácticas de distintas asignaturas de la titulación.

En cuanto al Aprendizaje-Servicio y prácticas vinculadas al territorio coincidimos con Traver-Martí, Moliner y Sales (2019) porque estas se ejecutan gracias a la labor de docentes de Educación Primaria. De cara a que el profesorado de infantil y secundaria 
también conociera y formara parte de dichos proyectos, sería imprescindible en las instituciones tejer proyectos con carácter humano no solo a nivel de etapa, sino de centro.

En conclusión, a pesar de las diferencias y similitudes que existen entre las estrategias metodológicas docentes, todo el profesorado tiene en común la lucha por las injusticias sociales y su esfuerzo por dotar a todas las personas de los conocimientos, las competencias y los valores necesarios que le permitan vivir con dignidad (Sanahuja, Moliner y Moliner, 2020; UNESCO, 2017). De este modo, en este estudio se reconoce el valor y las oportunidades que ofrece la diversidad en las prácticas, apostando por estrategias metodológicas flexibles, abiertas y sensibles que cuidan y empoderan las distintas formas de ser, pensar, sentir y actuar de los estudiantes.

\section{Limitaciones e investigaciones futuras}

La principal limitación de esta investigación es que todos los participantes eran de escuelas públicas y solo uno estaba desempañando su labor en un instituto privado. Desconocemos si estas mismas estrategias metodológicas se están llevando a cabo en instituciones concertadas y privadas, o existen otras distintas que pueden aportar al estudio y permiten la participación de todos los estudiantes.

Otra limitación es que el estudio se basa exclusivamente en entrevistas y no se han explorado estas estrategias metodológicas en la práctica diaria. Sin embargo, en una segunda fase de este estudio, pretendemos acceder al campo a través de la observación de las clases de los tres docentes más significativos de cada etapa educativa de cara a recoger datos desde la práctica. Asimismo, pretendemos que las voces de los estudiantes sean escuchadas. Para ello realizaremos entrevistas al alumnado que permitan complementar la información recogida previamente.

Por último, estudios futuros podrían centrarse en la formación del profesorado y en diseñar, desarrollar y evaluar programas de formación que ayudaran a equipos directivos, familias y docentes de diferentes etapas educativas a reflexionar y emprender prácticas más accesibles e inclusivas.

\section{Referencias}

Ainscow, M., Slee, R y Best, M. (2019). Editorial: La declaración de Salamanca 25 años después. Revista Internacional de Educación Inclusiva, 23(7-8), 671-676.

https://doi.org/10.1080/13603116.2019.1622800

Arnaiz, P. y Guirao, J. M. (2015). La autoevaluación de centros en España para la atención a la diversidad desde una perspectiva inclusiva. ACADI. Revista Electrónica Interuniversitaria de Formación del Profesorado, 18(1),45-101. https://doi.org/10.6018/reifop.18.1.214341

Azorín, C. y Sandoval, M. (2019). Apoyos para avanzar hacia una educación más inclusiva en los centros escolares: Análisis de guías para la acción. Siglo Cero, 50(3), 7-27. https://doi.org/10.14201/scero2019503727

Belavi, G. y Murillo, F. J. (2016). Educación, democracia y justicia social. Revista Internacional de Educación para la Justicia Social, 5(1), 13-34.

Black-Hawkins, K., Florian, L. y Rouse, M. (2007). Achievement and inclusion in schools. Routledge.

Crisol, E. (2019). Hacia una educación inclusiva para todos. Nuevas contribuciones. Profesorado, Revista de Currículum y Formación del Profesorado, 23(1), 1-9.

https://doi.org/10.30827/profesorado.v23i1.9155 
Cohen, L., Manion, L. y Morrison, K. (2000). Research methods in education. Routledge.

Day, C. (2019). Educadores comprometidos. Qué son, qué hace, por qué lo hacen y lo que verdaderamente importa. Narcea.

Díez-Palomar, J. y Flecha García, R. (2010). Comunidades de aprendizaje: Un proyecto de transformación social y educativa. Revista Interuniversitaria de Formación del Profesorado, 24(67), 19-30.

Florian, L. (2014). What counts as evidence of inclusive education? European Journal of Special Needs Education, 29(3), 286-294. https://doi.org/10.1080/08856257.2014.933551

Gale, T., Mills, C. y Cross, R. (2017). Socially inclusive teaching: Belief, design, actions as pedagogic work. Journal of Teacher Education, 68(3), 345-356. https://doi.org/10.1177/0022487116685754

Gonzalez-Gil, F., Martín-Pastor, E. y Poy, R. (2019). Educación inclusiva: Barreras y facilitadores para su desarrollo. Análisis de la percepción del profesorado. Profesorado. Revista de Currículum y Formación de Profesorado, 23(1), 243-263.

https://doi.org/10.30827/profesorado.v23i1.9153

Guerrero-Castañeda, B. J. (2016). Desafíos para la educación inclusiva en secundaria, de la utopía lo tangible. Revista Nacional e Internacional de Educación Inclusiva, 9(2), 87-103.

Harte, H. A. (2010). The project approach: A strategy for inclusive classrooms. Young Exceptional Children, 13(3), 15-27. https://doi.org/10.1177/1096250610364355

Henderson, M. C. y Lasley, E. (2014). Creating inclusive classrooms through the arts. Dimensions of Early Childhood, 42(3), 11-17.

Herrera, J. I., Parrilla, A., Blanco, A. y Guevara, G. (2018). La formación de docentes para la educación inclusiva. Un reto desde la Universidad Nacional de Educación en Ecuador. Revista Latinoamericana de Educación Inclusiva, $12(1), 2$ 1-38. https://doi.org/10.4067/S07 18-73782018000100021

Iglesias Varela, B., De la Madrid Heitzmann, L., Ramos Pérez, A., Robles Montes, C. y De Haro Martínez, A. S. (2013). Metodologías innovadoras e inclusivas en educación secundaria: Los grupos interactivos y la asamblea de aula. Tendencias Pedagógicas, 21, 63-78.

López-Melero, M. (2016). La LOMCE, un cuadro tenebroso que no responde a la diversidad. Revista Educación, Política y Sociedad, 1(2), 163-182.

López-Melero, M. (2018). Presentación. Educar como proceso de transformación en la convivencia. Revista Interuniversitaria de Formación del Profesorado, 92(32), 17-28.

Miles, M. y Huberman, A. (1994). Qualitative data analysis. Sage.

Moliner, O., Sanahuja, A. y Benet, A. (2017). Prácticas inclusivas en el aula desde la investigaciónacción. Colección Sapientia, 127, 35-56. https://doi.org/10.6035/Sapientia127

Muntaner, J. J. (2014). Prácticas inclusivas en el aula ordinaria. Revista Nacional e Internacional de Educación Inclusiva, 7(1), 1889-4208.

Muntaner, J. J., Rosselló, M. R. y De la Iglesia, B. (2016). Buenas prácticas en educación inclusiva. Educatio Siglo XXI, 34(1), 31-50. https://doi.org/10.6018/j/252521

Petrie, K., Devcich, J. y Fitzgerald, H. (2018). Working towards inclusive physical education in a primary school: Some days I just don't get it right. Physical Education and Sport Pedagogy, 23(4), 345-357. https://doi.org/10.1080/17408989.2018.1441391

Pujolàs Maset, P. (2002). Enseñar juntos a alumnos diferentes. La atención a la diversidad y la calidad en educación. Universidad de Vic. 
Rouse, M. (2009). Developing inclusive practice: A role for teachers and teacher education. Education in the North, 16, 6-13.

Sales, A., Moliner, O., Amiama, J. F. y Lozano, J. (2018). Escuela incluida. Revista Mexicana de Investigación Educativa, 23(77), 433-458.

Sanahuja, A., Moliner, O. y Moliner, L. (2020). Inclusive and democratic practices in primary school classrooms: A multiple case study in Spain. Educational Research, 62(1), 111-127. https://doi.org/10.1080/00131881.2020.1716631

Santamaría, I. y Stuardo, M. (2018). Una mirada a prácticas docentes desde un marco de justicia social. Revista Internacional de Educación para la Justicia Social, 7(1), 177-196. https://doi.org/10.15366/riejs2018.7.1.009

Sargeant, J. y Gillett-Swan, J. K. (2019). Voice-inclusive practice (VIP): A charter for authentic student engagement. International Journal of Children Rights, 7(1), 122-139. https://doi.org/10.1163/15718182-02701002

Sharma, U. y Jacobs, D. K. (2016). Predicting in-service educators' intentions to teach in inclusive classrooms in India and Australia. Teaching and Teacher Education, 55, 13-23. https://doi.org/10.1016/j.tate.2015.12.004

Simón, C., Echeita, G. y Sandoval, M. (2018). La incorporación de la voz del alumnado a la lesson study como estrategia de formación docente y mejora para la inclusión. Cultura y Educación, 3O(1), 215-225. https://doi.org/10.1080/11356405.2017.1416741

Souto-Manning, M. (2017). Generative text sets: Tools for negotiating critically inclusive early childhood teacher education pedagogical practices. Journal of Early Childhood Teacher Education, 38(1), 79-101. https://doi.org/10.1080/10901027.2016.1165760

Spratt, J. y Florian, L. (2015). Inclusive pedagogy: From learning to action. Supporting each individual in the context of everybody. Teaching and Teacher Education, 49, 89-96.

https://doi.org/10.1016/j.tate.2015.03.006

Stake, R. (2006). Multiple case study analysis. The Guilford.

Tjernberg, C. y Mattson, E. H. (2014). Inclusion in practice: A matter of school culture. European Journal of Special Needs Education, 29(2), 247-256. https://doi.org/10.1080/08856257.2014.891336

Traver-Martí, J., Moliner, O. y Sales, A. (2019). Negociando el currículum: Aprendizaje-servicio en la escuela incluida. Alteridad, 14(2),195-206. https://doi.org/10.17163/alt.v14n2.2019.04

UNESCO. (2017). Guía para asegurar la inclusión y la equidad en la inclusión. UNESCO.

Zhu, J., Li, H. y Hsieh, W. Y. (2019). Implementing inclusive education in an early childhood setting: A case study of a Hong Kong kindergarten. Early Child Development and Care, 189(2), 207-2 19. https://doi.org/10.1080/03004430.2017.1307841

\section{Breve CV de las autoras}

\section{Inmaculada Orozco}

Contratada predoctoral FPU del departamento de Didáctica y Organización Educativa de la Universidad de Sevilla. Su foco de estudio se centra en la pedagogía inclusiva en diferentes etapas educativas, desde Educación Infantil hasta Universidad. Entre sus líneas de investigación sobresalen temáticas como la diversidad, la inclusión educativa, la 
formación del profesorado y la investigación cualitativa. Ha publicado en revistas nacionales e internacionales y participado en distintos congresos internacionales. ORCID ID: https://orcid.org/o000-0001-5170-7805. Email: iorozco@us.es

\section{Anabel Moriña}

Profesora del Departamento de Didáctica y Organización Educativa de la Universidad de Sevilla. Su trayectoria profesional está vinculada a la educación inclusiva, exclusión educativa, formación continua del profesorado, discapacidad y Enseñanza Superior. Es coordinadora del Máster Universitario en Necesidades Educativas Especiales y Atención a la Diversidad en la Escuela de la Universidad de Sevilla. ORCID ID: https://orcid.org/0000-0002-0852-7523. Email: anabelm@us.es 